\title{
Interferometer for Testing Extended Surfaces Such as Surface Plates and Precision Ways
}

\author{
J. B. Saunders and John V. McDermott
}

(January 21, 1964)

\begin{abstract}
A modification of the previously described "Interferometer for Large Surfaces" is described that permits the use of smaller prisms for a given sensitivity and a more practical arrangement of the optics. This arrangement allows for better structural connection of the elements into a rigid and moveable unit. This is obtained by a 90 degree rotation of the prism relative to the plane of incidence of the light.
\end{abstract}

Since publishing a description of the interferometer for large surfaces ${ }^{1}$ these authors have considered means of facilitating its construction and applications. The modified version permits the use of a smaller prism for attaining the same sensitivity. The elements are more easily combined (mechanically) into a rigid unit.

Figure 1 is a view of the optical elements. Light from a monochromatic source is collimated before entering the plane entrance face of a Kösters prism. The beam is divided by amplitude division at the semireflecting dividing plane. After total internal reflection inside the prism the two component beams emerge parallel and normal to the prism face. One component beam, after emergence from the Kösters prism, is reflected normally from a mirror so that it returns along its path of incidence to the dividing plane. The other component beam is deviated by a plane wedge so that it is incident onto the surface to be tested. A second mirror receives this beam and returns it (after a second reflection from the surface being tested) along its previous path to the beam divider. The two component beams recombine at the beam divider and after a second division a component of each beam is received by the observer.

If the two paths are approximately equalized interference fringes of good contrast are observed.

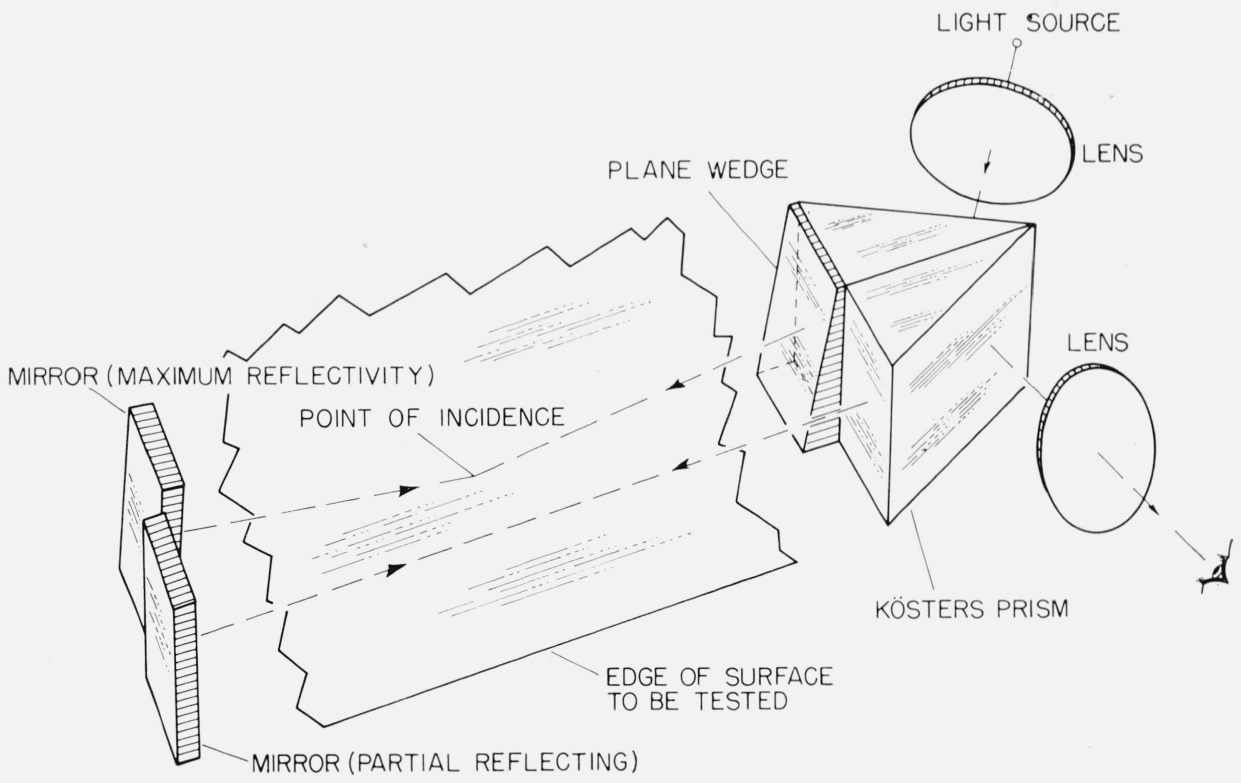

Figure 1. A plan view showing the arrangements of elements for a compact surface plate interferometer.

1 An interferometer for testing large surfaces, by James B. Saunders and Franz L. Gross, J. Res. NBS 62 (Apr. 1959) RP2943. 


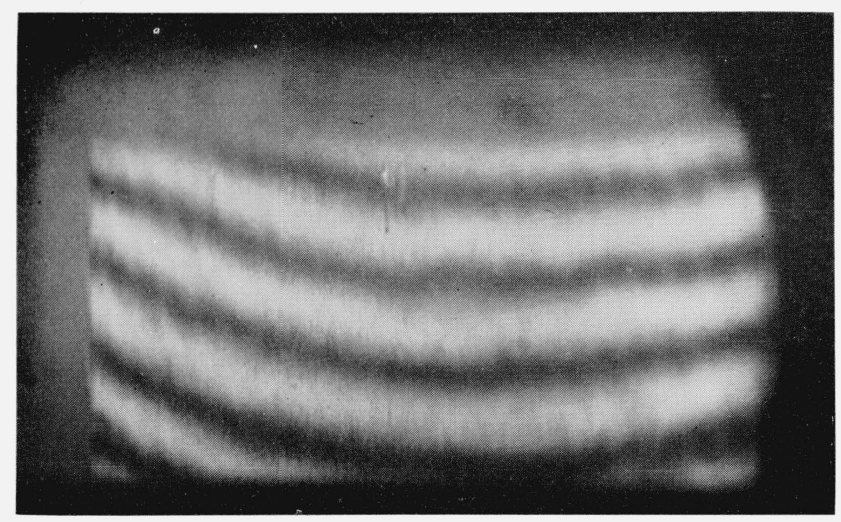

FiguRE 2. Interference fringes with light reflected from a 24 in. black granite surface plate.

The width and orientation of the fringes may be altered at will by adjusting either of the two mirrors. Figure 2 show fringes from a 24 in. surface plate of black granite.

Since the reflectivities of all surfaces to be tested are well below unity one mirror must have a higher reflectivity if the two recombined beams are to be approximately equal.
When testing large surfaces of the type indicated, one is usually concerned with measurement of the surface along a line. Consequently, the horizontal width of the aperture (or field of interference) can be relatively small. The height (fig. 1) of the prism determines the length of surface that may be tested with a given sensitivity (angle of incidence). Thus, a prism with a horizontal cross section that is small, relative to its height, can be used. This reduces the difficulty of constructing a prism having a given vertical aperture.

It will be noted that all elements of this interferometer are above the plane of the test surface. The mirrors are placed laterally to each other instead of one vertically above the other, as in the previously described instrument (see footnote 1). These differences allow for simplification of the mechanics of the instruments. The sensitivity may be altered by replacing the plane wedge with another having a different deviation and then readjusting the distance between mirrors and prism.

The sensitivity of this interferometer is the same as that described (see footnote 1). One fringe corresponds to a departure from flatness of $\lambda /(4 \cos \alpha)$, where $\lambda$ is the wavelength and $\alpha$ the angle of incidence.

(Paper 68C2-153) 